\title{
Rheumatoid Arthritis Induced by Botulinum Toxin Type A: A Case Report and Review of the Literature
}

\author{
Yanyan $G^{1,2 \#}$, Yupeng $L^{1 \#}$, Yuanyuan $L^{1,3}$, Fangfang \\ $\mathrm{Z}^{1,3}$ and Meiying $\mathrm{W}^{1 *}$ \\ ${ }^{1}$ Department of Rheumatology and Immunology, \\ Shenzhen Second People's Hospital, The First Affiliated \\ Hospital of Shenzhen University, Shenzhen, China \\ ${ }^{2}$ Department of Nephrology, Peking University Shenzhen \\ Hospital, Shenzhen, China \\ ${ }^{3}$ Department of Rheumatology and Immunology, Peking \\ University Shenzhen Hospital, Shenzhen, China \\ "Contributed equally to this paper \\ *Correspondling author: Meiying Wang, Department \\ of Rheumatology and Immunology, Shenzhen Second \\ People's Hospital, The First Affiliated Hospital of \\ Shenzhen University, Shenzhen 518035, China
}

Received: April 21, 2021; Accepted: May 15, 2021; Published: May 22, 2021

\begin{abstract}
Introduction: Botulinum Toxin Type A (BoNT/A) is a bacterial toxin commonly used in cosmetic therapy. Although there has been a great deal of clinical and basic research on the potential therapeutic applications of botulinum toxin there are few reports on its clinical toxicity and side effects.

Patient Concerns: A previously healthy 26-year-old woman developed joint pain and redness in her right toe, swelling in the posterior left foot and the interphalangeal joint of the right index finger, occasional shoulder pain, and morning stiffness for 30 minutes daily, 6 months after BoNT/A injection.

Diagnosis: Laboratory testing showed elevated Rheumatoid Factor (RF), anti-cyclic citrullinated peptide (anti-CCP), C-Reactive Protein (CRP), Erythrocyte Sedimentation Rate (ESR). Doppler ultrasound examination of the right hand and both feet showed synovial hyperplasia of the right wrist, right second proximal interphalangeal joint, both ankles, and right first metatarsophalangeal joint, as well as bony erosion in a left intertarsal joint. Magnetic Resonance (MR) examination for right hand showed multiple joint changes of right hand and wrist, which included synovium thickening and enhancement. She was diagnosed as rheumatoid arthritis.
\end{abstract}

Interventions: BoNT/A injection was stopped. Methotrexate 10mg was given once weekly and hydroxychloroquine $0.2 \mathrm{~g}$ was given twice daily with subsequent remission of arthritis.

Outcomes: At 12 months from diagnosis, patient reported complete joints remission.

Conclusions: This is the first report of rheumatoid arthritis caused by botulinum toxin injection, and we speculate on the mechanism of its occurrence in this paper. In addition, we systematically introduce the latest research results on the development mechanism of BoNT/A causing RA. This review suggests that it is necessary to further explore the specific mechanism of RA or osteochondral injury caused by BoNT/A, which will not only help to improve the current understanding of the potential toxic and side effects of BoNT/A in clinical application, but also promote the standardization of clinical application of BONT/A.

Keywords: Botulinum toxin type A; Rheumatoid arthritis; Immunoinflammatory response; Osteoclast; Osteochondral lesion

\section{Introduction}

In the mid and late $20^{\text {th }}$ century, doctors and researchers found that non-accommodative strabismus could be corrected by intramuscular injection of BoNT/A without surgery. Since then, further application and development of BoNT/A ensued. From its initial approval for the treatment of specific dystonia to its subsequent application in cosmetic indications, BoNT/A has gradually produced many other BoNT/A "biomimetic drugs" and innovative formulations. It is also playing an important role in the field of biological defense [1].

Clostridium botulinum is a gram-positive anaerobic bacterium with four phenotypic groups and genotypic lineages (I-IV). The toxin it produces is called botulinum toxin. At present, seven different immune serotypes of botulinum toxin have been identified, and different phenotypic groups produce different botulinum toxins: group I produces toxin $\mathrm{A}$ and $\mathrm{B}$, group II produces toxin $\mathrm{B}, \mathrm{E}$ and $\mathrm{F}$, and group III produces toxin $\mathrm{C}$ and $\mathrm{D}$. Among them, BoNT/A, BoNT/B, BoNT/E and BoNT/F can cause diseases in humans. BoNT/C and BoNT/D are usually associated with poultry or other animal poisoning. BoNT/D is usually inactive in human muscle, so it is often used as a targeted biological agent for mediating chronic diseases. Presently, BoNT/A is the most frequently used Botox in clinic [2].

BoNT/A is a kind of protein toxin, which is composed of a 100 $\mathrm{kDa}$ heavy chain and a $50 \mathrm{kDa}$ light chain through a disulphide bond to form a double-stranded polypeptide. Its heavy chain has the dual role of receptor binding and translocation, while the light chain is a zinc endopeptidase that can prevent the fusion of acetylcholinecontaining vesicles with the presynaptic membrane of motor neurons.
Austin J Orthopade \& Rheumatol - Volume 8 Issue 1 - 2021 ISSN: 2472-369X | www.austinpublishing group.com Meiying et al. (C) All rights are reserved
Citation: Yanyan G, Yupeng L, Yuanyuan L, Fangfang Z and Meiying W. Rheumatoid Arthritis Induced by Botulinum Toxin Type A: A Case Report and Review of the Literature. Austin J Orthopade \& Rheumatol. 2021; 8(1): 1096. 
It is precisely because of the role of light chain that the toxin can block nerve transmission and eventually lead to relaxing muscle paralysis and achieve its therapeutic effect in clinic [3].

BoNT/A currently has a variety of clinical applications: cosmetic therapy, cervical dystonia, severe primary axillary hyperhidrosis, strabismus, neurogenic detrusor hyperactivity, chronic migraine, upper limb spasm, blepharospasm, psoriasis, and it can reduce sebum production in the treatment of acne [4]. In addition, BoNT/A can be used to treat persistent pain caused by simple arthritis [5].

There are also a variety of BoNT/A products on the market: American products Abotulinumtoxin A, British DySPORT, Chinese BTXA (also known as "Hengli" toxin), other kinds of RimabotulinumtoxinB, incobotulinumtoxinA amongst others. Different product parameters can have differences in pharmacokinetics, pharmacodynamics, clinical efficacy and tolerance, including immunogenicity. At present, standardized titer analysis has not been used to evaluate product parameters. This may be a potential factor of adverse reactions caused by BoNT/A [1]. There are also case reports about the comprehensive complications caused by BoNT/A, the mechanism of which may be related to the immune inflammatory response induced by BoNT/A [6,7]. However, there are few cases which have been reported about the relationship of BoNT/A and joints. Here, we report a case of rheumatoid arthritis which is caused by BoNT/A.

\section{Case Presentation}

A previously healthy 26-year-old woman developed joint pain and redness in her right toe 6 months after BoNT/A injection. She also experienced painful swelling of the posterior left foot which worsened with prolonged ambulation. Arthritis symptoms did not improve after resting for 1 month and progressed to include painful swelling in the interphalangeal joint of the right index finger, occasional shoulder pain, and morning stiffness for 30 minutes daily. The woman had no history of trauma, fever, rash, oral ulcer, or Raynaud's phenomenon.

Laboratory testing showed elevated Rheumatoid Factor (RF) 239kU/L (normal: <20kU/L), anti-Cyclic Citrullinated Peptide (anti-CCP) 39.6U/mL (normal: $<5 \mathrm{U} / \mathrm{mL}$ ), C-Reactive Protein (CRP) $12.10 \mathrm{mg} / \mathrm{L}$ (normal: $<5 \mathrm{mg} / \mathrm{L}$ ), Erythrocyte Sedimentation Rate (ESR) $70 \mathrm{~mm} /$ hour (normal: $<20 \mathrm{~mm} /$ hour), Anti-Streptolysin O (ASO) $171 \mathrm{kU} / \mathrm{L}$ (normal: <200kU/L), and Antinuclear Antibody (ANA) was 1:320. The proportion of Regulatory $\mathrm{T}$ cells/T helper cells (Treg/Th) was significantly lower than the normal value of 0.5 . Doppler ultrasound examination of the right hand and both feet showed synovial hyperplasia of the right wrist, right second proximal interphalangeal joint, both ankles, and right first metatarsophalangeal joint, as well as bony erosion in a left intertarsal joint (Figure 1). Magnetic Resonance (MR) examination for right hand showed multiple joint changes of right hand and wrist, which included synovium thickening and enhancement (Figure2). She was diagnosed as rheumatoid arthritis. Methotrexate $10 \mathrm{mg}$ was given once weekly and hydroxychloroquine $0.2 \mathrm{~g}$ was given twice daily with subsequent remission of arthritis. At 12 months from diagnosis, patient reported complete joints remission.

\section{Discussion}

\section{Prevalence and presentation}

We conducted a literature search in PubMed from 1990 to 2020 for all the cases of adverse reactions caused by BoNT/A. The cases of adverse reactions associated with BoNT/A are listed in Table 1. We found only 1 report of joints beings affected after BoNT/A administration, which involved the temporomandibular joint [8]. Only one longitudinal study reported bone volume changes induced by BoNT/A. The remaining reports were not related to joints and bones [9-19]. To our knowledge, we are reporting the first case of rheumatoid arthritis caused by BoNT/A.
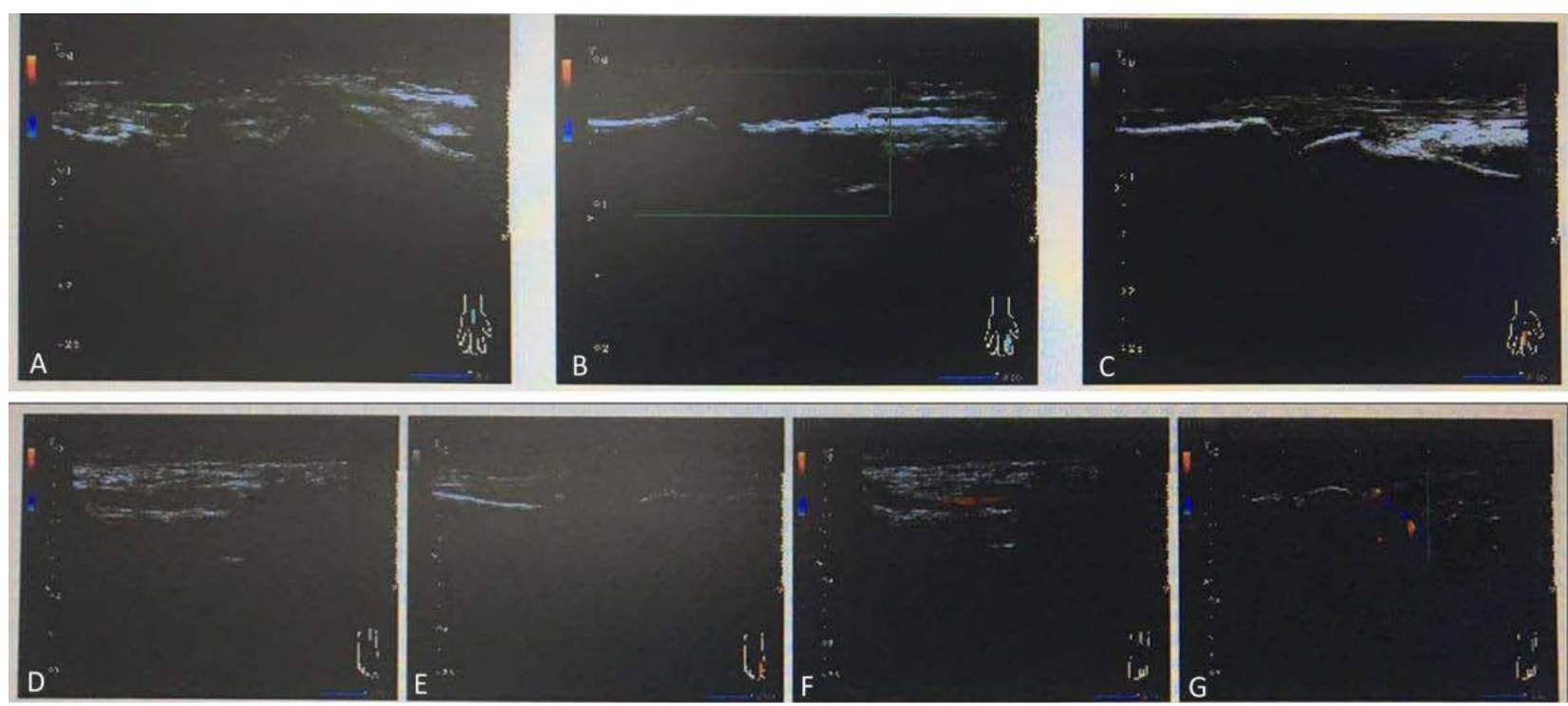

Figure 1: Ultrasound imaging presentation: A presents synovial proliferation in the right wrist; $B$ presents synovial proliferation in the PIP $2^{\text {nd }}$ of right hand; $C$ presents synovial proliferation in the PIP $3^{\text {rd }}$ of right hand; D presents synovial proliferation in the left ankle; F presents synovial proliferation in the right ankle; and $\mathrm{G}$ presents synovitis in the right intertarsal joints along with bone erosions. 
Table 1: Summary of prior reports of adverse reactions caused by BoNT/A.

\begin{tabular}{|c|c|c|c|c|c|c|c|}
\hline Author & Year & $\begin{array}{c}\text { No. of } \\
\text { case }\end{array}$ & Joint affected & Symptom & Imagining & Treatment & follow-up \\
\hline $\begin{array}{l}\text { Jeremie } \\
\text { D.Oliver }\end{array}$ & 2018 & 1 & no & decreasing pulmonary function & normal & stop injecting BoNT/A & 8 months \\
\hline Rashid Eama & 2018 & 9 & $\begin{array}{c}\text { muscle } \\
\text { weakness }\end{array}$ & $\begin{array}{c}\text { difficulty breathing, nausea and } \\
\text { vomiting }\end{array}$ & normal & stop injecting BoNT/A & $\begin{array}{l}6 \text { weeks-3 } \\
\text { months }\end{array}$ \\
\hline I.J. Moon & 2016 & 1 & no & angio-oedema and anaphylaxis & normal & symptomatic treatment & 4 hours \\
\hline Sasajima H. & 2017 & 1 & no & severe acute anterior uveitis & normal & scheduled infliximab injection & 3 months \\
\hline Phothong W & 2017 & 1 & no & dysphagia & normal & stop injecting BoNT/A & 3 months \\
\hline Dolar Bilge A & 2017 & 1 & no & diplopia & normal & stop injecting BoNT/A & 2-2.5 months \\
\hline Pisani LR & 2015 & 1 & no & Mondor's disease & normal & enoxaparin & 2 weeks \\
\hline Skaf GS & 2012 & 1 & no & $\begin{array}{l}\text { pseudoaneurysm of the superficial } \\
\text { temporal artery }\end{array}$ & $\mathrm{MRI}, \mathrm{CT}$ & endovascular therapy & 1 week \\
\hline Burguera JA & 2000 & 1 & no & polyradiculoneuritis & MRI & $\begin{array}{l}\text { intravenous highdose IgG and two times } \\
\text { of pulse therapy }\end{array}$ & 2 months \\
\hline Haug BA & 1990 & 1 & no & polyradiculoneuritis & normal & unknow & 2 months \\
\hline Aziz J & 2017 & 1 & yes & resorption of the mandibular condyle & MRI & unknow & unknow \\
\hline Lee $\mathrm{HJ}$ & 2017 & $\begin{array}{l}\text { a clinical } \\
\text { trial }\end{array}$ & yes & bony changes & СT & unknow & 6 months \\
\hline
\end{tabular}

Abbreviations: BoNT/A: Botulinum Toxin A; CT: Computed Tomography; MRI: Magnetic Resonance Imaging.

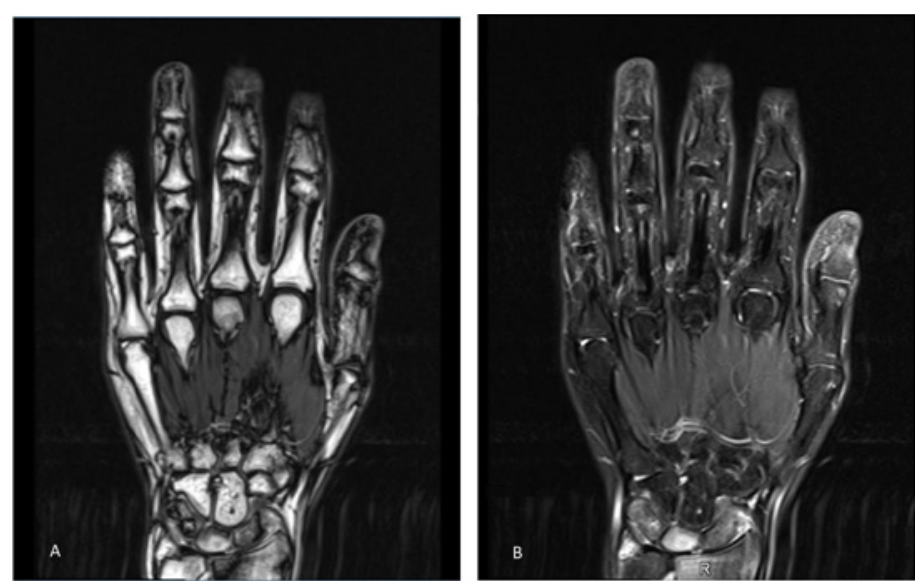

Figure 2: Magnetic Resonance Imaging (MRI) presentations of right wrist: A presents T1-weighted MRI, B presents T2-weighted MRI. In the right wrist, the navicular, the lunate and the triangular have long signals on the T1- and T2-weighted images, with soft tissue swelling. Partial enhancing was presented by the contrast enhanced MRI scans. Irregular thickening of synovium was shown in the right wrist, and significantly irregular enhancement was seen under the contrast enhanced MRI scans.

\section{Diagnosis and treatment}

It was difficult to confirm that the exact cause for all the cases is BoNT/A, with just depending on the use of BoNT/A before the disease. Thus diagnoses for all the cases were difficult. But considering the occurrence of adverse effect after BoNT/A administration and the resolution with withdrawal of BONTA and/or disease-specific treatment, it was reasonable for the authors to determine the BoNT/A adverse effect and make the diagnoses that those diseases were related with BONT/A. When the diagnoses were made, treatments were given to the patients. Four cases stopped injecting BoNT/A $[12,13,15,19]$, and one case reduced BoNT/A quantity [18]. Other therapies for those diseases caused by BoNT/A included symptomatic treatment, scheduled infliximab injection, enoxaparin, endovascular therapy, and intravenous high dose IgG and two times of pulse therapy. Three cases did not present their therapies. Nearly all the patients completely recovered in follow-up. Our case report is the first case of rheumatoid arthritis caused by BoNT/A, and the patient in our case recovered with methotrexate and hydroxychloroquine. We further explored mechanisms of BoNT/A side effects.

\section{Potential mechanisms for diseases caused by BoNT/A}

BONT/A induced inflammatory response: BONT/A may induce an inflammatory response in two separate ways. First, it can up-regulate genes such as TLR2, TNF, iNOS, CCL4, SLPI, stx11 and IRG1, which are related to cellular signal transduction, immunity and defense, protein metabolism and modification, neuronal activity, intracellular protein transport and muscle contraction. Under stimulation of BoNT/A, pro-inflammatory mediators such as Nitric Oxide (NO) and $\mathrm{TNF}^{\alpha}{ }^{\alpha}$ were released in a dose-dependent manner, which was dependent on the TLR2 pathway and regulated by $\mathrm{p} 38$ Mitogen-Activated Protein Kinase (MAPK), c-jun N-terminal kinase (JNK) and extracellular signal-regulated kinase (ERK) pathways [3]. Alternatively, BoNT/A may be involved in the process of inducing lysosomal dysfunction, which could aggravate the inflammatory 
response [20].

BoNT/A caused bone and cartilage damage: Studies have demonstrated BoNT/A can lead to bone and cartilage damage. Animal studies showed that BoNT/A could significantly decrease the level of serum calcium and increase the levels of serum phosphorus, C-Reactive Protein (CRP), alkaline phosphatase and tumor necrosis factor- $\alpha$ (TNF- $\alpha)$ in mice. After injection of BoNT/A into rats, histological analysis showed thinning of trabeculae of femoral dense bone and cancellous bone, as well as increased numbers of adipocytes in adjacent bone marrow [21]. Antonios et al found that unilateral injection of BoNT/A could cause paralysis within a few days, and significant ipsilateral trabecula loss [22]. Clinical studies have also demonstrated bone and cartilage changes. For example, BoNT/Ainduced masticatory muscle atrophy can promote mandibular loss [23]. Wang et al also discovered that injecting BoNT/A into paraspinal muscles could lead not only to muscle atrophy and dysfunction, but also to local bone loss and quality loss of the lumbar spine [24].

Underlying mechanisms of bone loss related to BoNT/A injection have been studied in detail. Intramuscular injection of BoNT/A increased local mRNA levels of inflammatory molecules, proteases, lipokines and mesenchymal stem cells, which may affect muscle repair. Fatty infiltration of injected muscle may further hinder muscle repair. After injection of BoNT/A, the contralateral muscles of mice also showed fibrosis, and these sites were not injected with BoNT/A, which was consistent with previous studies results that inflammation could also occur far from the treatment site of BoNT/A, but no evidence of fat infiltration was found in the contralateral muscles [25]. Hart et al found that regardless of the frequency of BoNT/A injection, the muscle mass and function of mice did not change or recover after 6 months. BoNT/A was currently thought to be effective for about 6 months to 1 year [26]. In the case mentioned in our article, the young woman also developed RA six months after the injection of BoNT/A. Although the effect on muscles after injection of BoNT/A was significant, it was worth noting that muscle and bone could interact with each other. During the study of botulinum toxininduced muscle paralysis in mice, Gatti et al found that after injection of BoNT/A, the porosity of muscle vessels increased, while the density of bone cell lacunae in the metaphyseal cortex of the tibia decreased. They speculated that this might be due to changes in fluid flow between tissues that affect molecular transport, resulting in changes in the mechanical signal transmission of bone cells, and the change also accelerated the occurrence of osteoporosis [27]. Moreover, Ausk et al found that inflammatory cells infiltrated with TNF- $\alpha$ and $\mathrm{IL}^{-1}$ increased within 24 hours after paralysis, bone marrow osteoclasts fused and the expression of pro-osteoclast inflammatory genes were up-regulated at 72 hours after BoNT/A injection. These changes allowed the bone marrow to form larger and more nuclear osteoclasts [28]. This explained that the interaction between muscle and bone at the cellular level played a key role in intraosseous homeostasis. These studies suggested the potential side effects or unexpected sequelae of intramuscular injection of BoNT/A on muscle and bone.

In conclusion, although adverse reactions caused by BoNT/A are rare, we report a case of RA induced by BoNT/A for the first time. The above mechanism studies help us to better understand the pathogenesis of RA, and injection of BoNT/A may induce bone and joint injury, and further expand the immune inflammatory response, resulting in rheumatoid arthritis. Its specific mechanism needs to be further studied.

\section{Acknowledgement}

We thank Dr. Daniel Kuo's assistance in editing the manuscript.

\section{Fundling Support}

This work was supported in part by grants from the Science and Technology Program for Basic Research in Shenzhen (No. JCYJ2019080909581124, No. JCYJ20170818153602439)

\section{Patient Consent and Confidentiality}

The patient signed a written informed consent form for the purpose of publication of results of this case study.

\section{References}

1. Tang M, Meng J, Wang J. New Engineered-Botulinum Toxins Inhibit the Release of Pain-Related Mediators. International Journal of Molecular Sciences. 2020; 21.

2. Mukund K, Ward SR, Lieber RL, Subramaniam S. Co-Expression Network Approach to Studying the Effects of Botulinum Neurotoxin-A. IEEE/ACM Trans Comput Biol Bioinform. 2018; 15: 2009-2016.

3. Kim YJ, Kim JH, Lee KJ, Choi MM, Kim YH, Rhie GE, et al. Botulinum neurotoxin type A induces TLR2-mediated inflammatory responses in macrophages. PLoS One. 2015; 10: e120840.

4. Monheit GD, Pickett A. AbobotulinumtoxinA. A 25-Year History. Aesthetic Surgery Journal. 2017; 37: S4-S11.

5. Singh JA, Mahowald ML. Intra-articular botulinum toxin A as an adjunctive therapy for refractory joint pain in patients with rheumatoid arthritis receiving biologics: a report of two cases. Joint Bone Spine. 2009; 76: 190-194.

6. Rouientan A, Alizadeh $\mathrm{OH}$, Mahmoudvand H, Tizmaghz A. Rare Complication of Botox Injection: A Case Report. World J Plast Surg. 2019; 8: 116-119.

7. Ricciardi L, Bove F, Fasano A. Xeomin((R)) use in patients with systemic immune reactions to other botulinum toxins type A. European Journal of Neurology. 2013; 20: e45-e46.

8. Aziz J, Awal D, Ayliffe P. Resorption of the mandibular condyle after injections of botulinum toxin A. Br J Oral Maxillofac Surg. 2017; 55: 987-988.

9. Burguera JA, Villaroya T, Lopez-Alemany M. Polyradiculoneuritis after botulinum toxin therapy for cervical dystonia. Clinical Neuropharmacology. 2000; 23: 226-228.

10. Wipf M, Palmowski-Wolfe A. [Therapeutic Use of Botulinum Toxin to Correct Misalignment of the Eyes]. Klin Monbl Augenheilkd. 2016; 233: 375-380.

11. Haug BA, Dressler D, Prange HW. Polyradiculoneuritis following botulinum toxin therapy. Journal of Neurology. 1990; 237: 62-63.

12. Oliver JD, Boesch RP, Mack KJ. Decreased Pulmonary Function During Botulinum Toxin A Therapy for Chronic Migraines in a 17-Year-Old Female. Headache. 2018; 58: 1259-1261.

13. Phothong W, Wanitphakdeedecha R, Keskool P, Manuskiatti W. A case of dysphagia following botulinum toxin injection for neck rejuvenation. J Cosmet Dermatol. 2017; 16: 15-17.

14. Pisani LR, Bramanti $P$, Calabro RS. A case of thrombosis of subcutaneous anterior chest veins (Mondor's disease) as an unusual complication of botulinum type A injection. Blood Coagul Fibrinolysis. 2015; 26: 685-686.

15. Rashid E, El-Mahdy NM, Kharoub HS, Gouda AS, EINabarawy NA, Megarbane B. latrogenic Botulism Outbreak in Egypt due to a Counterfeit Botulinum Toxin A Preparation - A Descriptive Series of Patient Features and Outcome. Basic Clin Pharmacol Toxicol. 2018; 123: 622-627.

16. Skaf GS, Domloj NT, Salameh JA, Atiyeh B. Pseudoaneurysm of the superficial temporal artery: a complication of botulinum toxin injection. Aesthetic Plast Surg. 2012; 36: 982-985. 
17. Sasajima H, Yagi S, Osada H, Zako M. Botulinum toxin-induced acute anterior uveitis in a patient with Behcet's disease under infliximab treatment: a case report. J Med Case Rep. 2017; 11: 124.

18. El-Heis S, Burke G, Gibb W, Ardern-Jones MR. Myaesthenia gravis exacerbation caused by axillary injection of botulinum toxin $A$ for treatment of hyperhidrosis. Clinical and Experimental Dermatology. 2017; 42: 357-359.

19. Dolar BA, Sadigov F, Salar-Gomceli S. Sixth nerve palsy following botulinum toxin injection for facial rejuvenation. Cutaneous and Ocular Toxicology. 2017; 36: 193-194.

20. Bonam SR, Wang F, Muller S. Lysosomes as a therapeutic target. Nature Reviews Drug Discovery. 2019; 18: 923-948.

21. Ali DM, Abdelzaher WY, Abdel-Hafez S. Evaluation of the rivastigmine role against botulinum toxin-A-induced osteoporosis in albino rats: A biochemical, histological, and immunohistochemical study. Human \& Experimental Toxicology. 2018; 37: 1323-1335.

22. Aliprantis AO, Stolina M, Kostenuik PJ, Poliachik SL, Warner SE, Bain SD, et al. Transient muscle paralysis degrades bone via rapid osteoclastogenesis. FASEB JOURNAL. 2012; 26: 1110-1118.

23. Balanta-Melo J, Toro-Ibacache V, Kupczik K, Buvinic S. Mandibular Bone Loss after Masticatory Muscles Intervention with Botulinum Toxin: An Approach from Basic Research to Clinical Findings. Toxins (Basel). 2019; 11: 84
24. Wang X, Wang S, Yan P, Bian Z, Li M, Hou C, et al. Paravertebral injection of botulinum toxin-A reduces lumbar vertebral bone quality. Journal of Orthopaedic Research. 2018; 36: 2664-26670.

25. Hart DA, Fortuna R, Herzog W. Messenger RNA profiling of rabbit quadriceps femoris after repeat injections of botulinum toxin: Evidence for a dynamic pattern without further structural alterations. Muscle \& Nerve. 2018; 57: 487493.

26. Awad F, Smith CD. Botox in reverse: a useful adjunct for acromial fractures. Ann R Coll Surg Engl. 2019; 101: 75-76.

27. Gatti V, Ghobryal B, Gelbs MJ, Gerber MB, Doty SB, Cardoso L, et al. Botoxinduced muscle paralysis alters intracortical porosity and osteocyte lacunar density in skeletally mature rats. Journal of Orthopaedic Research. 2019; 37: 1153-1163.

28. Ausk BJ, Worton LE, Smigiel KS, Kwon RY, Bain SD, Srinivasan S, et al. Muscle paralysis induces bone marrow inflammation and predisposition to formation of giant osteoclasts. Am J Physiol Cell Physiol. 2017; 313: C533-C540. 